\title{
Regulation of Myocardial Cell Growth and Death by the Hippo Pathway
}

\author{
Shohei Ikeda, MD, PhD; Junichi Sadoshima, MD, PhD
}

\begin{abstract}
Stress in the heart causes loss of cardiomyocytes (CMs), the accumulation of which leads to heart failure, a major cause of clinical mortality. The improvement of CM survival and facilitation of CM regeneration are major goals in treatment for heart failure. The Hippo pathway is an evolutionarily conserved signaling mechanism that regulates organ size by controlling both apoptosis and cell proliferation. The main components of the Hippo pathway, including Mst1/2, Lats1/2 and Yes-associated protein (Yap), are present in the mammalian heart and play an important role in regulating the growth and death of CMs. Recent research in the cardiac field has demonstrated that Yap, a key downstream transcriptional cofactor in the Hippo signaling pathway, plays a crucial role in regulating survival and proliferation/hypertrophy of CMs. Increasing lines of evidence suggest that Yap promotes regeneration of the heart after myocardial infarction. In this review, we summarize the current knowledge regarding the roles and functions of the Hippo pathway in the heart, with a particular emphasis on the role of Yap in regulating growth and death of CMs. (Circ J 2016; 80: 1511-1519)
\end{abstract}

Key Words: Apoptosis; Cardiac hypertrophy; Hippo signaling; Signal transduction; Yap

$\mathbf{H}$ eart failure (HF) is a critical risk factor for cardiac death, which is a major cause of human mortality in developed countries. ${ }^{1,2}$ Cardiac stress, including myocardial ischemia-reperfusion (IR) and high blood pressure, promotes the death of cardiomyocytes (CMs), resulting in decreases in the total number of CMs and cardiac dysfunction. Among the signaling mechanisms regulating the death of $\mathrm{CMs}$, the Hippo signaling pathway plays an important role in the progression of HF by regulating growth and death of CMs. ${ }^{3-5}$ This pathway, originally identified in Drosophila, is evolutionarily conserved from Drosophila to mammals, ${ }^{6}$ and generally serves as a key regulator of organ size, a function that is mediated through regulation of both apoptosis and cell proliferation.

A major function of the Hippo pathway is mediated through the Yes-associated protein (Yap), a transcription cofactor. Activation of the Hippo pathway promotes phosphorylation and consequent inactivation of Yap, thereby leading to cell apoptosis. In contrast, Yap activation leads to suppression of cell apoptosis and enhances cell survival and proliferation. Recent studies demonstrate that Yap plays a critical role not only in cardiac development but also in the control of cardiac function in the postnatal/adult heart. ${ }^{4-7-10}$ The fact that the presence of endogenous Yap is critical also in the adult heart ${ }^{4}$ is interesting because Yap induces cell proliferation in other cell types, whereas adult CMs do not proliferate efficiently. This review provides an overview of current findings about the role and the function of the Hippo-Yap pathway in the heart, with a particular emphasis on the role of Yap in mediat- ing survival and growth of CMs in the postnatal heart and the underlying signaling mechanisms mediating the function of Yap.

\section{Hippo Pathway Overview}

Components of the Hippo pathway were identified in Drosophila by genetic screens in 1995 . Two studies demonstrated that genetic deletion of Warts (wts) leads to a phenotype of tissue overgrowth in Drosophila. ${ }^{11,12}$ Subsequently, many studies have revealed that core components of the Hippo pathway are highly conserved in mammals (Table). Figure 1 shows a schematic representation of the Hippo pathway. Main components of the mammalian Hippo pathway include mammalian sterile 20-like kinase 1 and 2 (Mst1/2), large tumor suppressor 1 and 2 (Lats1/2), the scaffold protein Salvador (Sav, also known as WW domain-containing adaptor 45, or WW45), Mps one binder 1A and B (Mob1A/B), and Yap 1 (Yap)/ transcriptional coactivator with PDZ-binding motif (Taz), which are the mammalian orthologs of Drosophila hpo, wts, sav, mats, and yki, respectively.

Mst1/2 physically interacts with Sav1 and phosphorylates and activates Lats1/2. Recent evidence suggests that MAP4K family kinases act in parallel to Mst1/2 in HEK293A cells. ${ }^{13}$ However, the relative importance in CMs of MAP4Ks over Mst1/2 for activation of Lats 1/2 and suppression of Yap/Taz remains unknown. Lats $1 / 2$ phosphorylate and inactivate the transcriptional cofactor Yap by inducing its cytoplasmic translocation through 14-3-3 proteins and degradation through the

Received May 16, 2016; accepted May 17, 2016; released online June 10, 2016

Department of Cell Biology and Molecular Medicine, Rutgers - New Jersey Medical School, Newark, NJ, USA

Mailing address: Junichi Sadoshima, MD, PhD, Rutgers - New Jersey Medical School, Department of Cell Biology and Molecular

Medicine, 185 South Orange Avenue, MSB G-609, Newark, NJ 07103, USA. E-mail: sadoshju@ njms.rutgers.edu

ISSN-1346-9843 doi:10.1253/circj.CJ-16-0476

All rights are reserved to the Japanese Circulation Society. For permissions, please e-mail: cj@j-circ.or.jp 


\begin{tabular}{|llll|}
\hline \multicolumn{2}{|c|}{ Table. Components of the Hippo Pathway in Mammals and Drosophila } \\
Mammalian & \multicolumn{1}{c|}{ Drosophila } \\
MST 1/2 & Mammalian sterile 20-like kinase 1/2 & Hpo & Hippo \\
LATS $1 / 2$ & Large tumor suppressor kinase 1/2 & Wts & Warts \\
MOB 1A/1B & Mps one binder kinase activator-like 1A/1B & Mats & Mob as tumor suppressor \\
WW45/SAV 1 & Salvador homolog & Sav & Salvador \\
Yap & Yes-associated protein & Yki & Yorkie \\
Taz & Transcriptional coactivator with PDZ-binding motif & & \\
TEAD 1-4 & TEA domain family member 1-4 & Sd & Scalloped \\
\hline
\end{tabular}

\section{Hippo pathway - ON -}

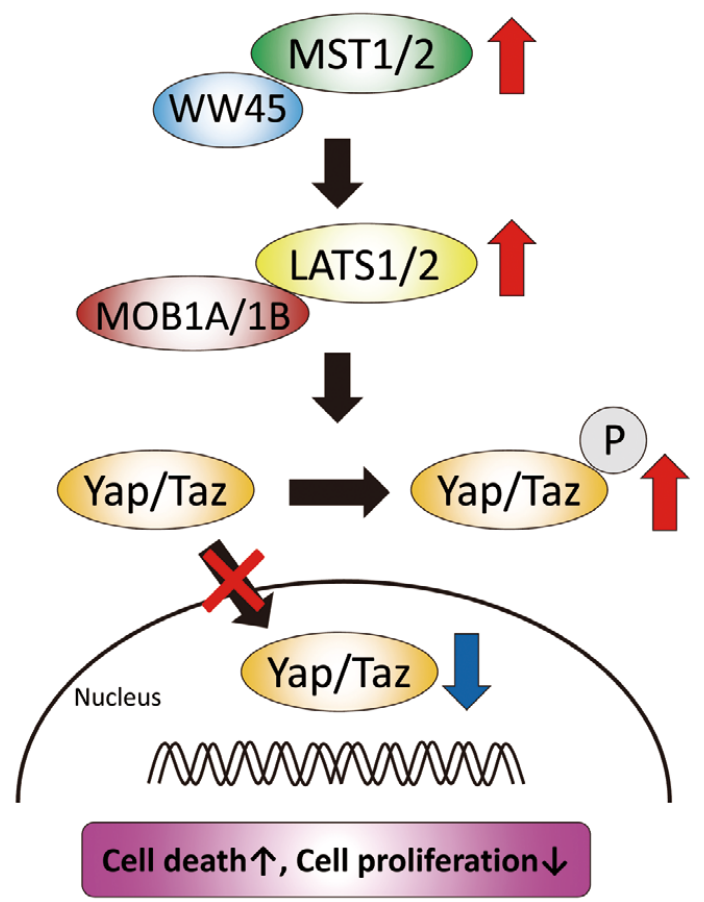

Hippo pathway - OFF -

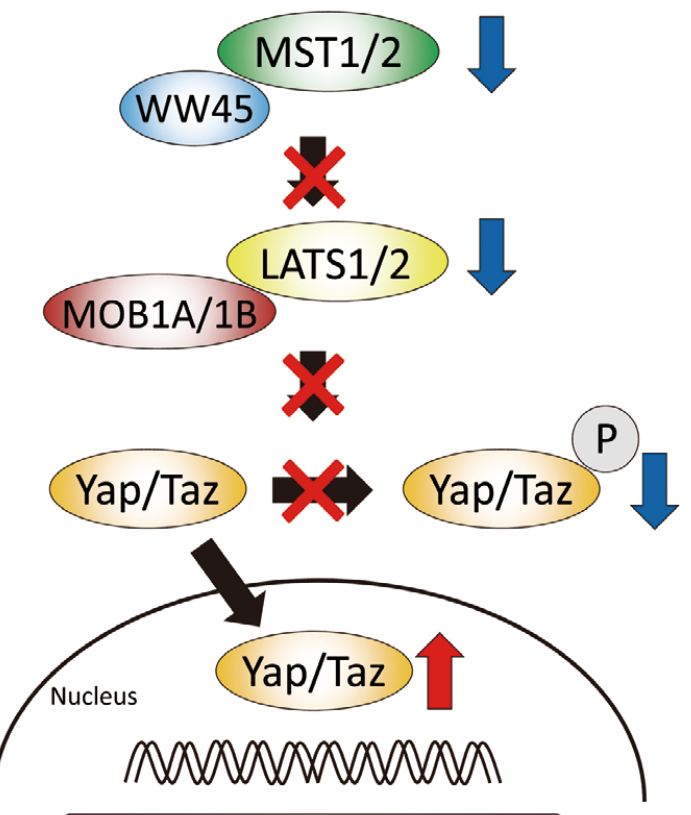

Cell death $\downarrow$, Cell proliferation $\uparrow$

Figure 1. Schematic representation of the canonical Hippo pathway components. The core components are Mst1/2, WW45, Lats1/2, Mob1, and Yap/Taz. Yap/Taz regulates gene expression, resulting in proliferation and survival. Lats1/2 phosphorylates and inhibits Yap/Taz. The scheme shows the consequences of activation and inactivation of the Hippo pathway in mammalian cells. Lats1/2, large tumor suppressor 1 and 2; Mob1A/1B, Mps one binder 1A and 1B; Mst1/2, mammalian sterile 20-like kinase 1 and 2; P, phosphoryl group; WW45, WW domain-containing adaptor 45, also known as Sav1, a Salvador scaffold protein homolog; Taz, transcriptional coactivator with PDZ-binding motif; Yap, Yes-associated protein.

ubiquitin proteasome system. ${ }^{14}$

When the Hippo pathway is inactivated, Yap translocates to the nucleus and interacts with multiple transcription factors, including the TEAD/TEF family transcription factors. ${ }^{15-18}$ The activity of Yap as a transcription cofactor is determined primarily by the level of Yap in the nucleus. In addition, vestigiallike family member 4 (VGLL4), a mammalian ortholog of Tondu-domain-containing growth inhibitor (Tgi), competes with Yap for TEAD binding, ${ }^{19,20}$ thereby serving as an endogenous inhibitor of Yap. TEAD regulates expression of genes involved in cell survival, proliferation, and metabolism, including connective tissue growth factor, cysteine-rich angio- genic inducer 61 (CYR61), axl receptor tyrosine kinase (AXL), amphiregulin (AREG), Myc, survivin (BIRC5) and angiomotin-like protein 2 (AMOTL2). ${ }^{21-25}$ The Yap-TEAD complex also recruits the NuRD transcriptional corepressor complex, thereby repressing target genes, including tumor-suppressor genes, such as DNA-damage-inducible transcript 4 (DDIT4) and TNF-related apoptosis-inducing ligand (Trail). ${ }^{26}$ Yap also interacts with myocardin-related transcription factor $\mathrm{A}$ (MRTA), a transcription cofactor, in response to sphingosine1-phosphate, thereby positively regulating transcription of CYR61 through cooperative actions between the Yap-TEAD pathway and the MRTA-serum response factor pathway. ${ }^{27}$ 


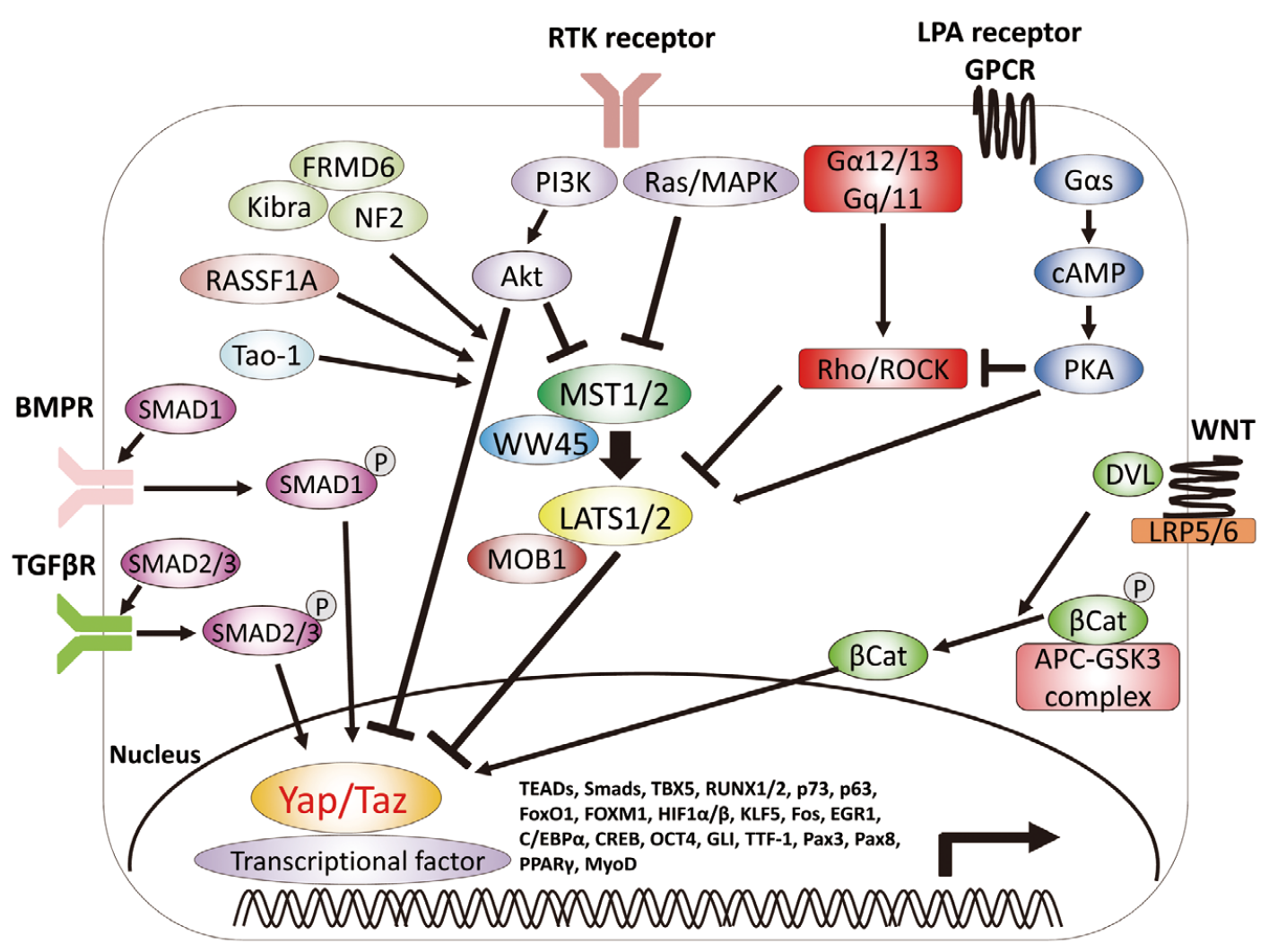

Figure 2. Schematic model of the Hippo pathway in mammals showing the signaling mechanisms modulating the activity of Yap/ Taz in mammalian cells. $\beta$ Cat, $\beta$ catenin; APC, adenomatous polyposis coli; BMPR, BMP receptor; C/EBPa, CCAAT/enhancerbinding proteins $\alpha$; CREB, CAMP responsive element binding protein; DVL, disheveled family proteins; EGR1, early growth response protein 1; FoxO1, forkhead box protein O1; FOXM1, forkhead box M1; FRMD6, FERM domain-containing protein 6; GPCR, G-protein-coupled receptor; GSK3, glycogen synthase kinase 3; HIF1a/ $\beta$, hypoxia inducible factor 1a/ $\beta$; KLF5, Kruppel-like factor 5; LPA, lysophosphatidic acid; LRP, lipoprotein receptor-related protein; OCT4, octamer-binding transcription factor 4; Pax, paired box; PI3K, phosphoinositide 3-kinase; PKA, protein kinase A; PPARY, peroxisome proliferator-activated receptor $Y$; RASSF1A, Ras-association domain family 1 isoform A; RTK, receptor tyrosine kinase; RUNX 1/2, Runt-related transcription factor 1/2; TBX5, T-box 5; TEAD, TEA domain transcription factor; TGF $\beta$ R, transforming growth factor- $\beta$ receptor; TTF- 1 , thyroid transcription factor-1.

Yap also interacts with other transcription factors, including TBX5, ${ }^{28,29}$ Smads, ${ }^{30-35}$ p63, ${ }^{36,37}$ p73, ${ }^{38-40}$ ErbB4, ${ }^{41}$ FoxO1, ${ }^{42}$ FOXM1, ${ }^{43}$ Runx 1/2, ${ }^{44-49}$ HIF1 $\alpha / \beta,{ }^{50,51}$ KLF5, ${ }^{52,53}$ Fos, ${ }^{54}$ EGR $1,{ }^{55,56} \mathrm{C} / \mathrm{EBP} \alpha,{ }^{57} \mathrm{CREB},{ }^{58}$ OCT4, ${ }^{59}$ GLI, ${ }^{60}$ TTF- $1,{ }^{61}$ Pax3, ${ }^{62}$ Pax $8,{ }^{63}$ PPAR $\gamma^{45}$ and MyoD,${ }^{64}$ thereby regulating a wide variety of genes (Figure 2).

In the adult heart, weak basal Mst1/2 and Lats1/2 activity allows only modest nuclear accumulation of Yap at baseline conditions. ${ }^{8}$ This poses the question of when and how Yap is activated in the adult heart. Because Mst1/2 and Lats1/2 are further activated by stress, it is important to know when these upstream kinases are inactivated in the heart. How the activity of Yap is regulated in CMs is poorly understood. Importantly, Yap can be regulated by upstream signaling mechanisms not traditionally included in the Hippo pathway. For example, mechanical forces, stiffness of extracellular matrixes, cell-cell contact, and cell adhesion regulate Yap through cellular tension, RhoA, and actin dynamics. ${ }^{65-68}$ Agonists for G protein-coupled receptors also regulate Yap through similar mechanisms. ${ }^{69} \mathrm{In}$ addition, it has recently been shown $\alpha$-catenin negatively regulates Yap, ${ }^{70}$ while S1P positively regulates Yap in CMs. ${ }^{4}$
There are many structural similarities between Yap and Taz (Figure 3). Although Yap cardiac-specific knockout (cKO) mice exhibit a significant cardiac phenotype, ${ }^{4}$ Taz cKO mice ${ }^{71}$ exhibit a normal cardiac phenotype and their survival curve appears identical to that of wild-type mice. Thus, it is possible that Yap can compensate for the loss of Taz but that Taz may not compensate for the loss of Yap. However, double cKO of Yap and Taz worsens the survival rate compared with Taz cKO and Yap cKO, suggesting that Yap and Taz also have non-overlapping functions. ${ }^{71}$ Alternatively, suppression of either Yap or Taz alone may not be sufficient to completely block their common downstream targets in the heart.

\section{Hippo Pathway in Heart Development}

The mammalian heart is the first organ formed during development. The heart-forming process from mesodermal tissues is precisely programmed and consists of forming a heart tube and heart loop architecture, which then progresses to 4-chamber components and an outflow tract. The molecular mechanisms regulating mammalian heart development remain to be 


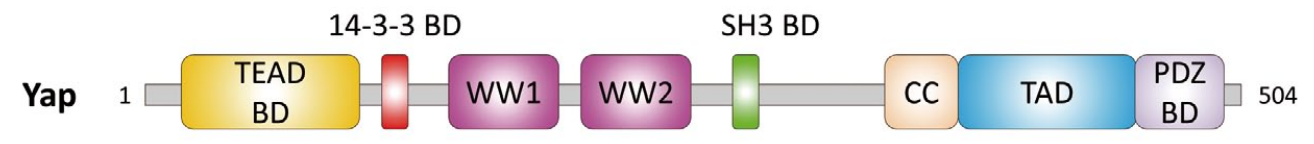

amino acids

14-3-3 BD

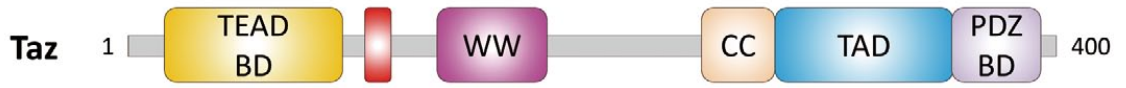

Figure 3. Schematic representation of the homologous structure of Yap and Taz. CC, coiled-coil domain; TAD, transactivation domain; TEAD BD, TEA domain family member binding domain.

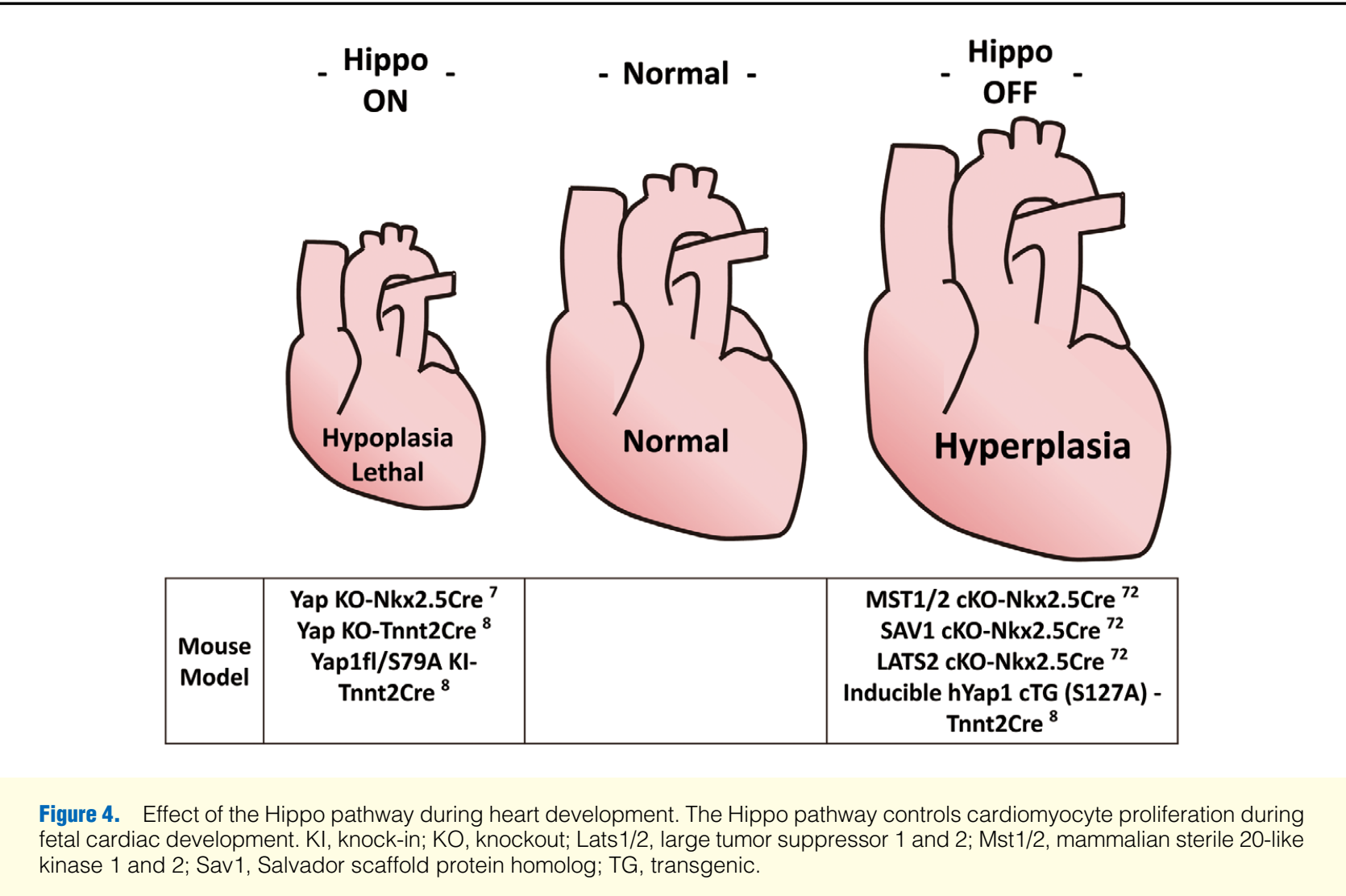

fully elucidated. However, it is known that the Hippo pathway plays a crucial role in regulating cardiac development. Mouse models of embryonic deletion of Sav1, Mst1/2 or Lats2 exhibit similar hyperplastic phenotypes with thickened ventricular walls. These mice die at early postnatal stages, with obvious heart enlargement. ${ }^{72}$ Interestingly, the Hippo pathway restrains CM proliferation and heart size by inhibiting Yap/Taz and the Wnt pathway during the development of the heart (Figure 4). Yap/Taz interacts with Tbx5, a key transcriptional factor regulating embryonic heart development. ${ }^{28}$ Yap inactivation during development leads to cardiac hypoplasia, causing lethality. ${ }^{7}$ Gain-of-function of Yap during development stimulates CM proliferation and enhances ventricular hypertrabeculation. Several genes containing the TEAD-binding MCAT motif are regulated by Yap. ${ }^{8}$ The MCAT motifs are located in the promoter-enhancer regions of muscle-specific genes, such as cardiac troponin T, $\beta$-myosin heavy chain, smooth muscle $\alpha$-actin, and skeletal $\alpha$-actin. TEADs play a crucial role in the regulation of these genes during development. ${ }^{73}$ TEAD1 disruption leads to heart defects and embryonic lethality in mice. ${ }^{74}$ On the other hand, TEAD1 overexpression in the postnatal mouse heart upregulates the fetal-type gene program and induces age-dependent dysfunction. ${ }^{75}$ These findings suggest that YapTEAD interaction is necessary for embryonic heart growth 
and that the appropriate control of Yap is necessary for the well-organized development of the heart. Interestingly, the heart size is normal in $\beta$-MHC-YapS112A transgenic mice, in which the transgene is expressed from E9, with decreases in CM size accompanied by increases in cell numbers. ${ }^{7}$ Taken together, the data suggest that Yap has several roles at each stage of heart development. These findings demonstrate the critical role of the Hippo pathway during cardiogenesis and suggest that appropriate levels of Yap activity are required for proper heart development.

\section{Myocardial Infarction//schemia-Reperfusion}

Loss of CMs is an important mechanism facilitating the development of HF. ${ }^{76}$ Mst1 is activated by pro-apoptotic stimuli and contributes to programmed cell death in cancer cell lines. ${ }^{77-81}$ Mst1 is one of the most strongly activated protein kinases, detected by in-gel myelin basic protein kinase assays, when CMs die by apoptosis. ${ }^{3}$ Cardiac-specific overexpression of Mst1 in transgenic mice induces elevated caspase activation and $\mathrm{CM}$ apoptosis, leading to dilated cardiomyopathy and premature death. ${ }^{3}$ Cardiac-specific overexpression of DNMst1 in mice protects the heart against IR injury and postmyocardial infarction (MI) cardiac remodeling. ${ }^{3}$

The Hippo pathway is highly compartmentalized in CMs. For example, IR activates Mst1 through a K-Ras-Rassf1Adependent mechanism in mitochondria, where Mst1 stimulates the mitochondrial mechanism of apoptosis by phosphorylating Bcl-xL. ${ }^{82}$ Mst1 activated through currently unknown mechanisms also phosphorylates Beclin 1 in the endoplasmic reticulum, thereby inhibiting autophagy. ${ }^{83}$ Because neither induction of apoptosis nor suppression of autophagy by these pathways requires either Lats2 or Yap, we have designated these as "non-canonical Hippo pathways". Cardiac stress also activates Mst1 and Lats2 through an NF2-dependent mechanism in the nucleus, where Lats2 induces nuclear exit of Yap (Matsuda et al, unpublished data). This pathway utilizes many components of the Hippo pathway, from NF2 to Yap, and, thus, it is designated as the "canonical Hippo pathway". Activation of the canonical Hippo pathway leads to stimulation of cell death and inhibition of compensatory hypertrophy by inhibiting Yap. ${ }^{4,10,42}$ Thus, depending upon the type of stress, Mst1 is activated in different subcellular spaces and mediates CM apoptosis through different mechanisms. Because the Hippo pathway in each subcellular compartment is activated by distinct mechanisms and targets distinct substrates, it is important to further clarify the specific molecular targets (cellular functions) regulated by the Hippo pathway in each subcellular compartment. Therapeutically, suppression of Mst1 may allow inhibition of Mst1 in multiple cellular compartments, thereby inhibiting the proapoptotic effects of Mst1 altogether.

Activation of the canonical Hippo pathway causes nuclear exit and downregulation of Yap. ${ }^{84}$ Deletion of Yap in the heart with tnnt-Cre, which allows downregulation of Yap during the fetal stage, causes lethal cardiac hypoplasia with low proliferative ability. In this model, the mice did not show significant differences in CM apoptosis. ${ }^{8}$ Interestingly, primarily postnatal deletion of Yap with $\alpha \mathrm{MHC}-\mathrm{Cre}$ induces robust increases in $\mathrm{CM}$ apoptosis, thereby inducing cardiomyopathy with premature death. ${ }^{4}$ Furthermore, a study using cardiac-specific Yap heterozygous KO mice generated with $\alpha \mathrm{MHC}-\mathrm{Cre}$ demonstrated significant increases in apoptosis after MI in adult mice. ${ }^{4}$ These findings suggest that endogenous Yap (manifested by loss-of-function studies) may have distinct roles in the heart before and after birth, namely, that it primarily affects cell proliferation during the fetal stage, but cell survival in the postnatal stage. Transgenic expression of activated Yap exhibited cardioprotective effects after MI. ${ }^{9}$ Similar findings were observed in Sav1 cKO mice, in which endogenous Yap is activated, after MI. ${ }^{85}$ In these models, increased proliferation of CMs was also observed in the heart. The relative importance of the pro-survival effect and cell-proliferative effects of overexpressed Yap in mediating cardioprotection in the post-MI heart remains to be clarified.

What is the downstream molecular mechanism mediating the pro-survival effects of Yap in the postnatal heart? At least 3 mechanisms are involved. First, Yap binds to FoxOs in the nucleus and promotes transcription of antioxidant genes, including catalase and manganese superoxide dismutase (MnSOD). ${ }^{42}$ Second, Yap promotes expression of miR-206 through stimulation of E-box binding transcription factors. miR-206 in turn suppresses forkhead bos P1 (FoxP1), a transcription factor that promotes death of CMs. ${ }^{10}$ Third, Yap promotes activation of Akt, a serine/threonine kinase that promotes cell survival, through unknown mechanisms stimulating PI3K.,9 The importance of each mechanism may differ depending upon the type of stress.

\section{Hypertrophy}

The Hippo pathway controls organ size by affecting both cell death and proliferation. An interesting question is whether activation and inactivation of the Hippo pathway affects the size of the adult mammalian heart, in which growth is regulated primarily by hypertrophy rather than proliferation.

Overexpression of Mst1 in the mouse heart induces chamber dilation and wall thinning of the left ventricle because of increased cell death. According to Laplace's law, dilation of the left ventricle with wall thinning increases wall stress, ${ }^{86}$ which normally stimulates hypertrophy as a compensatory mechanism in order to normalize the wall stress. However, mice with cardiac overexpression of Mst1 (Tg-Mst1) generated on the C57BL/6 background do not show significant increases in left ventricular weight/tibial length ratio at 3 months of age, and the longitudinal length of CMs isolated from the left ventricle is even smaller in Tg-Mst1 mice than in control mice, suggesting that compensatory hypertrophy is attenuated. ${ }^{3}$ We speculate that this contributes to the progressive deterioration of cardiac function in Tg-Mst1 mice, because the elevated wall stress increases oxygen consumption and cell death. Transgenic mice with cardiac-specific overexpression of Lats 2 also exhibit a dilated cardiomyopathy phenotype without cardiac hypertrophy that is similar to that of Tg-Mst1 mice. ${ }^{5}$ These results suggest that activation of the upstream kinases of the Hippo pathway negatively regulates cardiac hypertrophy. It would be interesting to test whether Mst1 and Lats2 also inhibit physiological hypertrophy, such as that induced by exercise.

Suppression of the upstream Hippo kinases alleviates Ser127 phosphorylation of Yap, thereby inducing nuclear accumulation of Yap. Overexpression of DN-Mst1 does not show any significant effect on cardiac function, chamber size or cardiac hypertrophy at baseline. ${ }^{3}$ However, $\alpha$-MHC-specific Tg-DNLATS2 mice show modest cardiac hypertrophy. ${ }^{5}$ In addition, human hypertrophic cardiomyopathy patients showed Yap activation in the heart. ${ }^{87}$ These results are consistent with the notion that inhibition of the Hippo pathway and activation of Yap may induce cardiac hypertrophy.

Interestingly, however, in both inducible cardiac-specific Lats1/2 KO mice and inducible cardiac-specific sav1 KO 
mice, the adult heart shows increases in CM proliferation but decreases in cardiac hypertrophy ${ }^{85}$ Similarly, miR-302/367 induces Yap activation through suppression of the core components of the Hippo pathways, thereby increasing cardiac proliferation in the adult heart. Importantly, these mice do not show cardiac hypertrophy. ${ }^{88}$ Thus, how suppression of the Hippo pathway affects the growth of the adult heart appears to be context-dependent even though the different interventions commonly stimulate Yap. It is possible that the extent of Yap activation may differ among different interventions. Furthermore, Mst1, Lats1 and Lats2 may have additional targets besides Yap. As we discuss later, the different interventions may allow Yap to interact with distinct transcription factors, such as TEAD and E-box binding transcription factors. Interestingly, regardless of the mode of cell growth, namely hypertrophy or proliferation, the adult mouse heart does not show obvious organ enlargement in response to inhibition of the Hippo pathway, a property that is distinct from that observed in several organs in Drosophila, mammalian liver, and the fetal heart, possibly because CM proliferation is inefficient in the adult heart.

It should be noted that although heterozygous Yap cKO mice have no obvious phenotype at baseline, they develop more severe cardiac dysfunction and dilation after MI ${ }^{4}$ Despite cardiac dysfunction and increases in the left ventricular wall stress caused by cardiac dilation, heterozygous Yap cKO mice do not exhibit enhancement of cardiac hypertrophy compared with wild-type mice, indicating that compensatory hypertrophy is suppressed when endogenous Yap is downregulated.

Although the role of Yap in cardiac hypertrophy is complex, we propose the following hypothesis. A certain level of Yap is required for compensatory hypertrophy in the presence of stress. Thus, loss of Yap function causes insufficient cardiac hypertrophy in response to stress, which leads to exacerbation of cardiac dysfunction. Overactivation of Yap, however, induces $\mathrm{CM}$ proliferation rather than hypertrophy. What is the underlying mechanism of the apparently dose-dependent function of Yap? Yap may regulate either hypertrophy or proliferation depending upon how it couples to downstream signaling mechanisms. For example, miR-206 mediates Yap-induced cardiac hypertrophy by downregulating FoxP $1 .{ }^{10}$ On the other hand, TEAD mediates proliferation rather than hypertrophy. In fact, overexpression of TEAD1 in the mouse heart rather inhibits hypertrophy. ${ }^{74}$ It is also possible that cross-talk between Yap and other signaling pathways regulating cell growth, such as Wnt ${ }^{7,57,72,89-91}$ and mTOR,,${ }^{92-94}$ may determine how Yap affects hypertrophy and proliferation of CMs under given conditions.

\section{Hippo Pathway in Heart Regeneration}

A low level of CM turnover is observed in the adult mammalian heart because of proliferation of existing CMs. ${ }^{95,96}$ Regeneration of damaged hearts may take place also through CM differentiation of cardiac stem cells. ${ }^{97,98}$ However, the adult mammalian heart obviously has insufficient ability to regenerate following injury, ${ }^{99}$ and the loss of CMs in response to stress leads to the development of HF. ${ }^{100}$ The Hippo pathway is known to play an important role in the regulation of tissue repair and regeneration in some organs. In particular, Yap promotes the ability to self-renew and differentiate in embryonic stem cells, ${ }^{101}$ satellite muscle cells ${ }^{102}$ and liver cells. ${ }^{103}$

Increased expression of Yap in the heart induces CM proliferation $^{5}$ and protects against $\mathrm{MI}$ in mice. ${ }^{71}$ Activation of endogenous Yap in the adult heart in Hippo-deficient mice, such as cardiac-specific Sav $1 \mathrm{KO}$ and cardiac-specific Lats1/2 $\mathrm{KO}$, also protects against MI ${ }^{85}$ Conversely, cardiac-specific deletion of Yap decreases CM proliferation after MI. ${ }^{8}$ In a mouse model of MI, Yap-positive CMs are observed in the peri-infarct area 3-5 days after initiation of MI. Because the Yap cKO mice had a larger scar area and developed more severe cardiac dysfunction, we speculate that Yap-positive CMs have salutary functions in the post-MI heart. Although the mechanism responsible for the greater infarct area in Yap cKO mice is currently unknown, endogenous Yap inhibits CM apoptosis and promotes $\mathrm{CM}$ proliferation in the peri-infarct area, thereby preventing expansion of MI.,71 Thus, endogenous Yap appears necessary for the post-MI recovery process. The mechanism by which Yap is upregulated in CMs in the peri-infarct area is currently unknown. Likewise, whether Yap-positive CMs originate from resident CMs or progenitor cells remains to be clarified. Hippo deficiency also enhances cardiac regeneration after cardiac apex resection and MI at postnatal day $8 .^{85}$ Further investigation is required to clarify whether the cardiac regeneration and functional recovery caused by Yap really achieves long-term functional recovery in post-MI hearts.

\section{Conclusions}

Increasing lines of evidence suggest that the Hippo pathway plays a critical role in regulating growth and death of CMs at the cell level, and myocardial injury after IR and the progression of HF in the heart at the organ level. Although the functional involvement of the Hippo pathway in cardiac pathology has become increasingly clear, many unanswered questions remains. These include but are not limited to the following. First, how is the activity of Yap regulated during cardiac hypertrophy and $\mathrm{HF}$ and what are the upstream signaling mechanisms? Although nuclear accumulation of Yap is inhibited in CMs at baseline, it can be induced in response to stress despite the fact that the Hippo pathway is generally activated by stress and inhibits the nuclear localization of Yap. Elucidating both Hippo pathway-dependent and -independent mechanisms regulating the nuclear localization of Yap in CMs is important. Second, is stimulation of CM proliferation by Yap really beneficial? Although a gain of Yap function promotes myocardial regeneration in the post-MI heart, $, 91,85,88$ its longterm effects and its effects in response to other types of stress, such as pressure overload, remain to be shown. Third, activation of Yap is observed in human patients with hypertrophic cardiomyopathy. ${ }^{87}$ In this condition, does inhibition of Yap ameliorate the progression of cardiomyopathy? Because Yap is involved in tumorigenesis, small molecule inhibitors of Yap are being developed. ${ }^{17,104-107}$ Whether inhibition of Yap alleviates cardiomyopathic growth remains to be tested. Fourth, Yap both positively and negatively regulates the activity of transcription factors. Thus, which transcription factor mediates the function of Yap in the heart during a given stress remains to be elucidated. Elucidating the detailed downstream signaling mechanisms of Yap may allow for the development of efficient treatments to protect the heart from stress. Fifth, the Hippo pathway may possess distinct functions in different cell types. ${ }^{69,108}$ The function of the Hippo pathway and Yap in the non-myocyte population in the heart remains to be elucidated. Finally, more study is needed to clarify both the overlapping and non-overlapping functions of Yap and Taz in the heart.

In summary, as prominent downstream effectors of the Hippo pathway, Yap/Taz appears to mediate many important 
functions in the heart. We believe that Yap/Taz are promising targets for the treatment of heart disease. Further investigation is required, however, in order to apply our knowledge regarding this signaling pathway to the clinical setting and achieve effective treatment of patients.

\section{Acknowledgments}

This work was supported in part by US. Public Health Service Grants HL67724, HL91469, HL102738, HL112330 and AG23039 (J.S.) and by the Leducq Foundation Transatlantic Network of Excellence (J.S.). S.I. has been supported by a Postdoctoral Fellowship from Japan Heart Foundation / Bayer Yakuhin Research Grant Abroad. We thank Dr H. Shimokawa at the Department of Cardiovascular Medicine, Tohoku University Graduate School of Medicine for his cooperation with S.I.'s Work in Rutgers University NJMS. We also thank Daniela Zablocki for assistance with the manuscript.

\section{References}

1. Roger VL. Epidemiology of heart failure. Circ Res 2013; 113: 646-659.

2. Sakata Y, Shimokawa H. Epidemiology of heart failure in asia. Circ J 2013; 77: 2209-2217.

3. Yamamoto S, Yang G, Zablocki D, Liu J, Hong C, Kim SJ, et al. Activation of mst 1 causes dilated cardiomyopathy by stimulating apoptosis without compensatory ventricular myocyte hypertrophy. J Clin Invest 2003; 111: 1463-1474.

4. Del Re DP, Yang Y, Nakano N, Cho J, Zhai P, Yamamoto T, et al. Yes-associated protein isoform 1 (yap1) promotes cardiomyocyte survival and growth to protect against myocardial ischemic injury. J Biol Chem 2013; 288: 3977-3988.

5. Matsui Y, Nakano N, Shao D, Gao S, Luo W, Hong C, et al. Lats2 is a negative regulator of myocyte size in the heart. Circ Res 2008; 103: $1309-1318$.

6. Zhou Q, Li L, Zhao B, Guan KL. The hippo pathway in heart development, regeneration, and diseases. Circ Res 2015; 116: $1431-1447$

7. Xin M, Kim Y, Sutherland LB, Qi X, McAnally J, Schwartz RJ, et al. Regulation of insulin-like growth factor signaling by yap governs cardiomyocyte proliferation and embryonic heart size. Sci Signal 2011; 4: ra70.

8. von Gise A, Lin Z, Schlegelmilch K, Honor LB, Pan GM, Buck JN, et al. Yap1, the nuclear target of hippo signaling, stimulates heart growth through cardiomyocyte proliferation but not hypertrophy. Proc Natl Acad Sci USA 2012; 109: 2394-2399.

9. Lin Z, von Gise A, Zhou P, Gu F, Ma Q, Jiang J, et al. Cardiacspecific yap activation improves cardiac function and survival in an experimental murine mi model. Circ Res 2014; 115: 354-363.

10. Yang Y, Del Re DP, Nakano N, Sciarretta S, Zhai P, Park J, et al. Mir-206 mediates yap-induced cardiac hypertrophy and survival. Circ Res 2015; 117: 891-904.

11. Justice RW, Zilian O, Woods DF, Noll M, Bryant PJ. The drosophila tumor suppressor gene warts encodes a homolog of human myotonic dystrophy kinase and is required for the control of cell shape and proliferation. Genes Dev 1995; 9: 534-546.

12. Harvey PA, Leinwand LA. The cell biology of disease: Cellular mechanisms of cardiomyopathy. J Cell Biol 2011; 194: 355-365.

13. Meng Z, Moroishi T, Mottier-Pavie V, Plouffe SW, Hansen CG, Hong AW, et al. Map4k family kinases act in parallel to mst $1 / 2$ to activate lats $1 / 2$ in the hippo pathway. Nat Commun 2015; 6: 8357.

14. Adler JJ, Johnson DE, Heller BL, Bringman LR, Ranahan WP, Conwell MD, et al. Serum deprivation inhibits the transcriptional co-activator yap and cell growth via phosphorylation of the 130-kda isoform of angiomotin by the lats1/2 protein kinases. Proc Natl Acad Sci USA 2013; 110: 17368-17373.

15. Zhao B, Ye X, Yu J, Li L, Li W, Li S, et al. Tead mediates yapdependent gene induction and growth control. Genes Dev 2008; 22: $1962-1971$.

16. Zhao B, Kim J, Ye X, Lai ZC, Guan KL. Both tead-binding and ww domains are required for the growth stimulation and oncogenic transformation activity of yes-associated protein. Cancer Res 2009; 69: $1089-1098$.

17. Liu-Chittenden Y, Huang B, Shim JS, Chen Q, Lee SJ, Anders RA, et al. Genetic and pharmacological disruption of the tead-yap complex suppresses the oncogenic activity of yap. Genes Dev 2012; 26: $1300-1305$

18. Marti P, Stein C, Blumer T, Abraham Y, Dill MT, Pikiolek M, et al. Yap promotes proliferation, chemoresistance, and angiogenesis in human cholangiocarcinoma through tead transcription factors. Hepatology 2015; 62: 1497-1510.

19. Jiao S, Wang H, Shi Z, Dong A, Zhang W, Song X, et al. A peptide mimicking vgll4 function acts as a yap antagonist therapy against gastric cancer. Cancer Cell 2014; 25: 166-180.

20. Zhang W, Gao Y, Li P, Shi Z, Guo T, Li F, et al. Vgll4 functions as a new tumor suppressor in lung cancer by negatively regulating the yap-tead transcriptional complex. Cell Res 2014; 24: 331-343.

21. Xu MZ, Chan SW, Liu AM, Wong KF, Fan ST, Chen J, et al. Axl receptor kinase is a mediator of yap-dependent oncogenic functions in hepatocellular carcinoma. Oncogene 2011; 30: 1229-1240.

22. Zhang J, Ji JY, Yu M, Overholtzer M, Smolen GA, Wang R, et al. Yap-dependent induction of amphiregulin identifies a non-cellautonomous component of the hippo pathway. Nat Cell Biol 2009; 11: $1444-1450$.

23. Dong J, Feldmann G, Huang J, Wu S, Zhang N, Comerford SA, et al. Elucidation of a universal size-control mechanism in drosophila and mammals. Cell 2007; 130: 1120-1133.

24. Lu L, Li Y, Kim SM, Bossuyt W, Liu P, Qiu Q, et al. Hippo signaling is a potent in vivo growth and tumor suppressor pathway in the mammalian liver. Proc Natl Acad Sci USA 2010; 107: 1437-1442.

25. Wang W, Huang J, Chen J. Angiomotin-like proteins associate with and negatively regulate yap $1 . J$ Biol Chem $2011 ; 286$ : 4364-4370.

26. Kim M, Kim T, Johnson RL, Lim DS. Transcriptional co-repressor function of the hippo pathway transducers yap and taz. Cell Rep 2015; 11: 270-282.

27. Yu OM, Miyamoto S, Brown JH. Myocardin-related transcription factor $\mathrm{A}$ and yes-associated protein exert dual control in $\mathrm{G}$ proteincoupled receptor- and RhoA-mediated transcriptional regulation and cell proliferation. Mol Cell Biol 2016; 36: 39-49.

28. Murakami M, Nakagawa M, Olson EN, Nakagawa O. A WW domain protein TAZ is a critical coactivator for TBX5, a transcription factor implicated in Holt-Oram syndrome. Proc Natl Acad Sci USA 2005; 102: $18034-18039$.

29. Rosenbluh J, Nijhawan D, Cox AG, Li X, Neal JT, Schafer EJ, et al. Beta-catenin-driven cancers require a yap1 transcriptional complex for survival and tumorigenesis. Cell 2012; 151: 1457-1473.

30. Ferrigno O, Lallemand F, Verrecchia F, L'Hoste S, Camonis J, Atfi A, et al. Yes-associated protein (YAP65) interacts with Smad7 and potentiates its inhibitory activity against TGF-beta/Smad signaling. Oncogene 2002; 21: 4879-4884.

31. Varelas X, Sakuma R, Samavarchi-Tehrani P, Peerani R, Rao BM, Dembowy J, et al. Taz controls smad nucleocytoplasmic shuttling and regulates human embryonic stem-cell self-renewal. Nat Cell Biol 2008; 10: 837-848.

32. Alarcon C, Zaromytidou AI, Xi Q, Gao S, Yu J, Fujisawa S, et al. Nuclear CDKs drive Smad transcriptional activation and turnover in BMP and TGF-beta pathways. Cell 2009; 139: 757-769.

33. Varelas X, Samavarchi-Tehrani P, Narimatsu M, Weiss A, Cockburn K, Larsen BG, et al. The Crumbs complex couples cell density sensing to Hippo-dependent control of the TGF-beta-SMAD pathway. Dev Cell 2010; 19: 831-844.

34. Fujii M, Toyoda T, Nakanishi H, Yatabe Y, Sato A, Matsudaira Y, et al. TGF-beta synergizes with defects in the Hippo pathway to stimulate human malignant mesothelioma growth. J Exp Med 2012; 209: $479-494$.

35. Narimatsu M, Samavarchi-Tehrani P, Varelas X, Wrana JL. Distinct polarity cues direct Taz/Yap and TGFbeta receptor localization to differentially control TGFbeta-induced Smad signaling. Dev Cell 2015; 32: 652-656.

36. Chatterjee A, Sen T, Chang X, Sidransky D. Yes-associated protein 1 regulates the stability of DeltaNp63alpha. Cell Cycle 2010; 9: $162-167$

37. Valencia-Sama I, Zhao Y, Lai D, Janse van Rensburg HJ, Hao Y, Yang X. Hippo component TAZ functions as a co-repressor and negatively regulates DeltaNp63 transcription through TEA domain (TEAD) transcription factor. J Biol Chem 2015; 290: 1690616917.

38. Strano S, Munarriz E, Rossi M, Castagnoli L, Shaul Y, Sacchi A, et al. Physical interaction with Yes-associated protein enhances p73 transcriptional activity. J Biol Chem 2001; 276: 15164-15173.

39. Basu S, Totty NF, Irwin MS, Sudol M, Downward J. Akt phosphorylates the Yes-associated protein, YAP, to induce interaction with 14-3-3 and attenuation of p73-mediated apoptosis. Mol Cell 2003; 11: $11-23$.

40. Lapi E, Di Agostino S, Donzelli S, Gal H, Domany E, Rechavi G, et al. PML, YAP, and p73 are components of a proapoptotic autoregulatory feedback loop. Mol Cell 2008; 32: 803-814.

41. Komuro A, Nagai M, Navin NE, Sudol M. WW domain-containing protein YAP associates with ErbB-4 and acts as a co-transcriptional 
activator for the carboxyl-terminal fragment of ErbB-4 that translocates to the nucleus. J Biol Chem 2003; 278: 33334-33341.

42. Shao D, Zhai P, Del Re DP, Sciarretta S, Yabuta N, Nojima H, et al. A functional interaction between Hippo-YAP signalling and FoxO1 mediates the oxidative stress response. Nat Commun 2014; 5: 3315 .

43. Eisinger-Mathason TS, Mucaj V, Biju KM, Nakazawa MS, Gohil M, Cash TP, et al. Deregulation of the Hippo pathway in soft-tissue sarcoma promotes FoxM1 expression and tumorigenesis. Proc Natl Acad Sci USA 2015; 112: E3402-E3411.

44. Yagi R, Chen LF, Shigesada K, Murakami Y, Ito Y. A WW domain-containing Yes-associated protein (YAP) is a novel transcriptional co-activator. EMBO J 1999; 18: 2551-2562.

45. Hong JH, Hwang ES, McManus MT, Amsterdam A, Tian Y, Kalmukova R, et al. Taz, a transcriptional modulator of mesenchymal stem cell differentiation. Science 2005; 309: 1074-1078.

46. Levy D, Adamovich Y, Reuven N, Shaul Y. Yap1 phosphorylation by c-Abl is a critical step in selective activation of proapoptotic genes in response to DNA damage. Mol Cell 2008; 29: 350-361.

47. Cui CB, Cooper LF, Yang X, Karsenty G, Aukhil I. Transcriptional coactivation of bone-specific transcription factor Cbfal by TAZ. Mol Cell Biol 2003; 23: 1004-1013.

48. Zaidi SK, Sullivan AJ, Medina R, Ito Y, van Wijnen AJ, Stein JL, et al. Tyrosine phosphorylation controls Runx2-mediated subnuclear targeting of YAP to repress transcription. EMBO J 2004; 23: $790-799$.

49. Ito Y, Bae SC, Chuang LS. The Runx family: Developmental regulators in cancer. Nat Rev Cancer 2015; 15: 81-95.

50. Bendinelli P, Maroni P, Matteucci E, Luzzati A, Perrucchini G, Desiderio MA. Hypoxia inducible factor-1 is activated by transcriptional co-activator with PDZ-binding motif (TAZ) versus WWdomaincontaining oxidoreductase (WWOX) in hypoxic microenvironment of bone metastasis from breast cancer. Eur J Cancer 2013; 49: $2608-2618$.

51. Ma B, Chen Y, Chen L, Cheng H, Mu C, Li J, et al. Hypoxia regulates Hippo signalling through the SIAH2 ubiquitin E3 ligase. Nat Cell Biol 2015; 17: 95-103.

52. Zhi X, Zhao D, Zhou Z, Liu R, Chen C. Yap promotes breast cell proliferation and survival partially through stabilizing the KLF5 transcription factor. Am J Pathol 2012; 180: 2452-2461.

53. Gao Y, Shi Q, Xu S, Du C, Liang L, Wu K, et al. Curcumin promotes KLF5 proteasome degradation through downregulating YAP/TAZ in bladder cancer cells. Int J Mol Sci 2014; 15: $15173-$ 15187.

54. Zhang W, Nandakumar N, Shi Y, Manzano M, Smith A, Graham $\mathrm{G}$, et al. Downstream of mutant KRAS, the transcription regulator YAP is essential for neoplastic progression to pancreatic ductal adenocarcinoma. Sci Signal 2014; 7: ra42.

55. Zagurovskaya M, Shareef MM, Das A, Reeves A, Gupta S, Sudol M, et al. EGR-1 forms a complex with YAP-1 and upregulates Bax expression in irradiated prostate carcinoma cells. Oncogene 2009; 28: $1121-1131$.

56. Nguyen LT, Tretiakova MS, Silvis MR, Lucas J, Klezovitch O, Coleman I, et al. ERG activates the YAP1 transcriptional program and induces the development of age-related prostate tumors. Cancer Cell 2015; 27: 797-808.

57. Wang J, Park JS, Wei Y, Rajurkar M, Cotton JL, Fan Q, et al. TRIB2 acts downstream of Wnt/TCF in liver cancer cells to regulate YAP and C/EBPalpha function. Mol Cell 2013; 51: 211-225.

58. Zhang T, Zhang J, You X, Liu Q, Du Y, Gao Y, et al. Hepatitis B virus $\mathrm{X}$ protein modulates oncogene Yes-associated protein by CREB to promote growth of hepatoma cells. Hepatology 2012; 56: 2051-2059.

59. Beyer TA, Weiss A, Khomchuk Y, Huang K, Ogunjimi AA, Varelas X, et al. Switch enhancers interpret TGF-beta and Hippo signaling to control cell fate in human embryonic stem cells. Cell Rep 2013; 5: 1611-1624.

60. Tariki M, Dhanyamraju PK, Fendrich V, Borggrefe T, Feldmann G, Lauth M. The Yes-associated protein controls the cell density regulation of hedgehog signaling. Oncogenesis 2014; 3: e112, doi:10.1038/oncsis.2014.27.

61. Park KS, Whitsett JA, Di Palma T, Hong JH, Yaffe MB, Zannini M. TAZ interacts with TTF-1 and regulates expression of surfactant protein-C. J Biol Chem 2004; 279: 17384-17390.

62. Murakami M, Tominaga J, Makita R, Uchijima Y, Kurihara Y, Nakagawa $\mathrm{O}$, et al. Transcriptional activity of Pax 3 is co-activated by TAZ. Biochem Biophys Res Commun 2006; 339: 533-539.

63. Di Palma T, D'Andrea B, Liguori GL, Liguoro A, de Cristofaro T, Del Prete D, et al. TAZ is a coactivator for Pax8 and TTF-1, two transcription factors involved in thyroid differentiation. Exp Cell
Res 2009; 315: $162-175$.

64. Jeong H, Bae S, An SY, Byun MR, Hwang JH, Yaffe MB, et al. TAZ as a novel enhancer of MyoD-mediated myogenic differentiation. FASEB J 2010; 24: 3310-3320.

65. Dupont S, Morsut L, Aragona M, Enzo E, Giulitti S, Cordenonsi M, et al. Role of YAP/TAZ in mechanotransduction. Nature 2011; 474: $179-183$.

66. Zhao B, Li L, Wang L, Wang CY, Yu J, Guan KL. Cell detachment activates the Hippo pathway via cytoskeleton reorganization to induce anoikis. Genes Dev 2012; 26: 54-68.

67. Sansores-Garcia L, Bossuyt W, Wada K, Yonemura S, Tao C, Sasaki $\mathrm{H}$, et al. Modulating F-actin organization induces organ growth by affecting the Hippo pathway. EMBO J 2011; 30: 23252335 .

68. Aragona M, Panciera T, Manfrin A, Giulitti S, Michielin F, Elvassore N, et al. A mechanical checkpoint controls multicellular growth through YAP/TAZ regulation by actin-processing factors. Cell 2013; 154: 1047-1059.

69. Yu FX, Zhao B, Panupinthu N, Jewell JL, Lian I, Wang LH, et al. Regulation of the Hippo-YAP pathway by G-protein-coupled receptor signaling. Cell 2012; 150: 780-791.

70. Li J, Gao E, Vite A, Yi R, Gomez L, Goossens S, et al. Alphacatenins control cardiomyocyte proliferation by regulating YAP activity. Circ Res 2015; 116: 70-79.

71. Xin M, Kim Y, Sutherland LB, Murakami M, Qi X, McAnally J, et al. Hippo pathway effector YAP promotes cardiac regeneration. Proc Natl Acad Sci USA 2013; 110: 13839-13844.

72. Heallen T, Zhang M, Wang J, Bonilla-Claudio M, Klysik E, Johnson RL, et al. Hippo pathway inhibits Wnt signaling to restrain cardiomyocyte proliferation and heart size. Science 2011; 332: $458-461$.

73. Yoshida T. MCAT elements and the TEF-1 family of transcription factors in muscle development and disease. Arterioscler Thromb Vasc Biol 2008; 28: 8-17.

74. Chen Z, Friedrich GA, Soriano P. Transcriptional enhancer factor 1 disruption by a retroviral gene trap leads to heart defects and embryonic lethality in mice. Genes Dev 1994; 8: 2293-2301.

75. Tsika RW, Ma L, Kehat I, Schramm C, Simmer G, Morgan B, et al. TEAD-1 overexpression in the mouse heart promotes an agedependent heart dysfunction. J Biol Chem 2010; 285: $13721-$ 13735.

76. Olivetti G, Abbi R, Quaini F, Kajstura J, Cheng W, Nitahara JA, et al. Apoptosis in the failing human heart. N Engl J Med 1997; 336: $1131-1141$.

77. Graves JD, Gotoh Y, Draves KE, Ambrose D, Han DK, Wright M, et al. Caspase-mediated activation and induction of apoptosis by the mammalian Ste20-like kinase Mst1. EMBO J 1998; 17: $2224-$ 2234.

78. Graves JD, Draves KE, Gotoh Y, Krebs EG, Clark EA. Both phosphorylation and caspase-mediated cleavage contribute to regulation of the Ste20-like protein kinase Mst1 during CD95/Fas-induced apoptosis. J Biol Chem 2001; 276: 14909-14915.

79. Lee KK, Yonehara S. Phosphorylation and dimerization regulate nucleocytoplasmic shuttling of mammalian Ste20-like kinase (Mst). J Biol Chem 2002; 277: 12351-12358.

80. Glantschnig H, Rodan GA, Reszka AA. Mapping of Mst1 kinase sites of phosphorylation: Activation and autophosphorylation. $J$ Biol Chem 2002; 277: 42987-42996.

81. Cheung WL, Ajiro K, Samejima K, Kloc M, Cheung P, Mizzen $\mathrm{CA}$, et al. Apoptotic phosphorylation of histone H2B is mediated by mammalian sterile twenty kinase. Cell 2003; 113: 507-517.

82. Del Re DP, Matsuda T, Zhai P, Maejima Y, Jain MR, Liu T, et al. Mst1 promotes cardiac myocyte apoptosis through phosphorylation and inhibition of Bcl-xl. Mol Cell 2014; 54: 639-650.

83. Maejima Y, Kyoi S, Zhai P, Liu T, Li H, Ivessa A, et al. Mst1 inhibits autophagy by promoting the interaction between beclin 1 and Bcl-2. Nat Med 2013; 19: $1478-1488$.

84. Wu S, Liu Y, Zheng Y, Dong J, Pan D. The TEAD/TEF family protein scalloped mediates transcriptional output of the Hippo growth-regulatory pathway. Dev Cell 2008; 14: 388-398.

85. Heallen T, Morikawa Y, Leach J, Tao G, Willerson JT, Johnson RL, et al. Hippo signaling impedes adult heart regeneration. Development 2013; 140: 4683-4690.

86. Goda A, Masuyama T. Left ventricular hypertrophy as a target of treatment in patients with hypertension. Circ J 2014; 78: $2633-$ 2634.

87. Wang P, Mao B, Luo W, Wei B, Jiang W, Liu D, et al. The alteration of Hippo/Yap signaling in the development of hypertrophic cardiomyopathy. Basic Res Cardiol 2014; 109: 435.

88. Tian Y, Liu Y, Wang T, Zhou N, Kong J, Chen L, et al. A 
microRNA-Hippo pathway that promotes cardiomyocyte proliferation and cardiac regeneration in mice. Sci Transl Med 2015; 7: 279ra38

89. Imajo M, Miyatake K, Iimura A, Miyamoto A, Nishida E. A molecular mechanism that links Hippo signalling to the inhibition of Wnt/ beta-catenin signalling. EMBO J 2012; 31: 1109-1122.

90. Azzolin L, Zanconato F, Bresolin S, Forcato M, Basso G, Bicciato S, et al. Role of TAZ as mediator of Wnt signaling. Cell 2012; 151: $1443-1456$.

91. Azzolin L, Panciera T, Soligo S, Enzo E, Bicciato S, Dupont S, et al. YAP/TAZ incorporation in the beta-catenin destruction complex orchestrates the Wnt response. Cell 2014; 158: 157-170.

92. Lee $\mathrm{CH}$, Inoki $\mathrm{K}$, Guan KL. mTOR pathway as a target in tissue hypertrophy. Annu Rev Pharmacol Toxicol 2007; 47: 443-467.

93. Tumaneng K, Schlegelmilch K, Russell RC, Yimlamai D, Basnet $\mathrm{H}$, Mahadevan N, et al. Yap mediates crosstalk between the Hippo and PI(3)K-TOR pathways by suppressing PTEN via miR-29. Nat Cell Biol 2012; 14: 1322-1329.

94. Lin Z, Zhou P, von Gise A, Gu F, Ma Q, Chen J, et al. Pi3kcb links Hippo-YAP and PI3K-AKT signaling pathways to promote cardiomyocyte proliferation and survival. Circ Res 2015; 116: 35-45.

95. Bergmann O, Bhardwaj RD, Bernard S, Zdunek S, Barnabe-Heider $\mathrm{F}$, Walsh S, et al. Evidence for cardiomyocyte renewal in humans. Science 2009; 324: 98-102.

96. Senyo SE, Steinhauser ML, Pizzimenti CL, Yang VK, Cai L, Wang $\mathrm{M}$, et al. Mammalian heart renewal by pre-existing cardiomyocytes. Nature 2013; 493: 433-436.

97. Nadal-Ginard B, Kajstura J, Leri A, Anversa P. Myocyte death, growth, and regeneration in cardiac hypertrophy and failure. Circ Res 2003; 92: 139-150.

98. Porrello ER, Mahmoud AI, Simpson E, Hill JA, Richardson JA, Olson EN, et al. Transient regenerative potential of the neonatal mouse heart. Science 2011; 331: 1078-1080.
99. Segers VF, Lee RT. Stem-cell therapy for cardiac disease. Nature 2008; 451: 937-942.

100. Waeber C, Walther T. Sphingosine-1-phosphate as a potential target for the treatment of myocardial infarction. Circ J 2014; 78: 795-802.

101. Lian I, Kim J, Okazawa H, Zhao J, Zhao B, Yu J, et al. The role of YAP transcription coactivator in regulating stem cell self-renewal and differentiation. Genes Dev 2010; 24: 1106-1118.

102. Judson RN, Tremblay AM, Knopp P, White RB, Urcia R, De Bari $\mathrm{C}$, et al. The Hippo pathway member YAP plays a key role in influencing fate decisions in muscle satellite cells. J Cell Sci 2012; 125: 6009-6019.

103. Yimlamai D, Christodoulou C, Galli GG, Yanger K, Pepe-Mooney B, Gurung B, et al. Hippo pathway activity influences liver cell fate. Cell 2014; 157: 1324-1338.

104. Liang N, Zhang C, Dill P, Panasyuk G, Pion D, Koka V, et al. Regulation of YAP by mTOR and autophagy reveals a therapeutic target of tuberous sclerosis complex. J Exp Med 2014; 211: $2249-2263$.

105. Zhang H, Ramakrishnan SK, Triner D, Centofanti B, Maitra D, Gyorffy B, et al. Tumor-selective proteotoxicity of verteporfin inhibits colon cancer progression independently of YAP1. Sci Signal 2015; 8: ra98.

106. Wang W, Li N, Li X, Tran MK, Han X, Chen J. Tankyrase inhibitors target YAP by stabilizing angiomotin family proteins. Cell Rep 2015; 13: 524-532.

107. Oku Y, Nishiya N, Shito T, Yamamoto R, Yamamoto Y, Oyama C, et al. Small molecules inhibiting the nuclear localization of YAP/ TAZ for chemotherapeutics and chemosensitizers against breast cancers. FEBS Open Bio 2015; 5: 542-549.

108. Yu FX, Guan KL. The Hippo pathway: Regulators and regulations. Genes Dev 2013; 27: 355-371. 